\title{
Distributionally Robust Co-Optimization of Energy and Reserve Dispatch of Integrated Electricity and Heat System
}

\author{
Skalyga, Mikhail; Wu, Quiwei
}

Published in:

Proceedings of 2020 International Conference on Probabilistic Methods Applied to Power Systems

Link to article, DOI:

10.1109/PMAPS47429.2020.9183678

Publication date:

2020

Document Version

Peer reviewed version

Link back to DTU Orbit

Citation (APA):

Skalyga, M., \& Wu, Q. (2020). Distributionally Robust Co-Optimization of Energy and Reserve Dispatch of Integrated Electricity and Heat System. In Proceedings of 2020 International Conference on Probabilistic Methods Applied to Power Systems (pp. 1-6). IEEE. https://doi.org/10.1109/PMAPS47429.2020.9183678

\section{General rights}

Copyright and moral rights for the publications made accessible in the public portal are retained by the authors and/or other copyright owners and it is a condition of accessing publications that users recognise and abide by the legal requirements associated with these rights.

- Users may download and print one copy of any publication from the public portal for the purpose of private study or research.

- You may not further distribute the material or use it for any profit-making activity or commercial gain

- You may freely distribute the URL identifying the publication in the public portal 


\section{Distributionally Robust Co-Optimization of Energy and Reserve Dispatch of Integrated Electricity and Heat System}

\author{
Mikhail Skalyga \\ Center for Electric Power and Energy \\ Technical University of Denmark \\ Kgs. Lyngby, Denmark \\ mikska@elektro.dtu.dk
}

\author{
Quiwei $\mathrm{Wu}$ \\ Center for Electric Power and Energy \\ Technical University of Denmark \\ Kgs. Lyngby, Denmark \\ qw@elektro.dtu.dk
}

\begin{abstract}
The combined operation of integrated energy systems is increasingly becoming a crucial topic for renewable energy dominated power systems operation. Flexibility from the district heating system could be used to deal with the uncertainty of renewable energy sources. We formulate a distributionally robust optimization problem for co-optimizing energy and reserve dispatch of the integrated electricity and heating system with a moment-based ambiguity set. The reserve allocation has been modeled through the participation vectors of the controllable generation units. The total reserve capacity has been defined implicitly and is a function of the uncertainty. The proposed model has been transformed into a second-order cone programming (SOCP) optimization problem by applying convex relaxation and linearization of the district heating network equations. Case studies on the integrated six-bus and seven-node system to demonstrate the efficacy of the proposed model.

Index Terms-Integrated energy systems, district heating, distributionally robust optimization, energy and reserve dispatch, convex relaxation, second-order cone programming
\end{abstract}

\section{INTRODUCTION}

According to the recently published study [1], one of the greatest challenges in the science of wind energy is the integration of wind power plans into the future electricity grid. In Denmark, the future primary goal is to achieve $100 \%$ renewable generation by 2050 [2]. Employing flexibility from the district heating system is one of the solutions among others to achieve this goal [3]. Optimal operation of the integrated energy systems and co-optimization of these systems is among the most discussed topics of these days. Several studies on this topic have been conducted so far. In [4], combined heat and power dispatch considering pipeline storage in the district heating network was performed. The model was formulated as a mixed-integer nonlinear programming (MINLP) optimization problem and solved with an iterative algorithm. The main shortfall of [4] is that the final solution is locally optimal. In [5], the optimal operation of the integrated electricity and heating system with a wind power plant was investigated. The final model is a large-scale non-linear program (NLP) that was solved with the decomposition coordinated algorithm. The equations given in [5] are accurate for describing an electrical system, but the final solution is again locally optimal.
To achieve a global optimum of the integrated system operation, the authors in [6], [7] applied convex relaxations of the highly nonlinear district heating network equations. In [6], the resulting model is a mixed-integer linear programming (MILP) problem which accounts for energy storage in the pipes. In [7], the authors introduced mixed-integer conic programming (MICP) formulation and the change in mass flow direction was considered. However, none of the above-mentioned studies managed renewable energy uncertainty.

Traditional approaches to deal with the uncertainty from the renewable energy sources are stochastic optimization (SO) and robust optimization (RO). In [8], the SO approach was applied to optimally schedule micro-grid generation units. A key problem of this approach is that for the large-sized systems, the amount of generated scenarios increases significantly and computational issue may arise. In contrast, the RO approach tends to obtain over-conservative solutions because the objective function minimizes the worst-case cost, which happens rarely. In [9], a robust unit commitment model with combined heat and power networks was developed. Since the mass flow rates were considered as fixed parameters, the resulting model is a two-stage MILP and was solved via the column-and-constraint generation (C\&CG) method. Distributionally robust optimization (DRO) approach could effectively overcome the downsides of SO and RO [10]. This innovative method represents the uncertainty through an ambiguity set, and the solution robust against all the possible distributions inside the ambiguity set. In [11], [12], the DRO approach has been successfully applied to the optimal power flow problems. In [13], energy and reserve dispatch with DRO joint chance constraints was studied. The ambiguity set was constructed with Wasserstein metric and tractable approximations were introduced.

The aim of this work is to extend the current knowledge of the operation of integrated electricity and district heating systems. The contributions of this study are summarized as follows:

- DRO formulation of energy and reserve dispatch of the combined electricity and heating system was introduced, 
with a moment-based ambiguity set. It requires the information only about the first and second moment of uncertainty.

- Non-linear district heating network equations were relaxed and the problem transformed into second-order cone programming (SOCP) optimization problem.

- The model was validated on a small study case in a datadriven manner.

This paper is organized as follows. In section II and III, district heating network (DHN) and power system model are presented. In IV, distributionally robust (DR) reformulation of the chance-constraints is introduced. Section $\mathrm{V}$ gives a brief overview of the convex relaxations of the DHN equations. In Section VI, a case study is analyzed. Our conclusions are drawn in Section VII.

\section{DISTRICT HEATING NETWORK MODEL}

Modeling of the district heating system is inspired by the work in [6]. The meaning of all variables used can be found in Online Appendix [14].

The continuity of flow is

$$
\begin{aligned}
& \sum_{p \in \mathcal{S}_{n}^{E}} m_{p}^{S}+m_{s}^{H S}=\sum_{p \in \mathcal{S}_{n}^{S}} m_{p}^{S}+m_{l}^{H L} \\
& \sum_{p \in \mathcal{S}_{n}^{E}} m_{p}^{R}+m_{s}^{H S}=\sum_{p \in \mathcal{S}_{n}^{S}} m_{p}^{R}+m_{l}^{H L}, \\
& \forall n \in \Lambda^{N}, s \in \Theta_{n}^{H S}, l \in \Theta_{n}^{H L}
\end{aligned}
$$

All mass flows are bounded by the operational limits

$$
\begin{aligned}
& \underline{m}_{p}^{S} \leq m_{p}^{S} \leq \bar{m}_{p}^{S}, \underline{m}_{p}^{R} \leq m_{p}^{R} \leq \bar{m}_{p}^{R}, \forall p \in \Lambda^{P} \\
& \underline{m}_{s}^{H S} \leq m_{s}^{H S} \leq \bar{m}_{s}^{H S}, \forall s \in \Theta_{n}^{H S} \\
& \underline{m}_{l}^{H L} \leq m_{l}^{H L} \leq \bar{m}_{l}^{H L}, \forall l \in \Theta_{n}^{H L}
\end{aligned}
$$

The pressure drops along the pipes due to the friction.

$$
\begin{aligned}
& p r_{n_{1}}^{S}-p r_{n_{2}}^{S}=K_{p}\left(m_{p}^{S}\right)^{2}, p r_{n_{1}}^{R}-p r_{n_{2}}^{R}=K_{p}\left(m_{p}^{R}\right)^{2}, \\
& \forall p \in \Lambda^{P}, n_{1} \in \Theta_{n}^{P, \text { OUT }}, n_{2} \in \Theta_{n}^{P, I N}
\end{aligned}
$$

The pressures are within the limits

$$
\underline{p r}_{n}^{S} \leq p r_{n}^{S} \leq \overline{p r}_{n}^{S}, \underline{p r}_{n}^{R} \leq p r_{n}^{R} \leq \overline{p r}_{n}^{R}, \forall n \in \Lambda^{N}
$$

More details of the pipe resistance coefficient calculation $K_{p}$ can be found in reference [15]. It is worth mentioning that if the network has a mesh structure and contains more than one loop, the loop pressure equation has to be added. The heat power output of heat sources is modeled as

$$
H_{s}^{H S}=C m_{s}^{H S}\left(T_{n}^{S}-T_{n}^{R}\right), \forall n \in \Lambda^{N}, \forall s \in \Theta_{n}^{H S}
$$

and limited as

$$
\underline{H}_{s}^{H S} \leq H_{s}^{H S} \leq \bar{H}_{s}^{H S}, \forall s \in \Theta_{n}^{H S}
$$

A circulation water pump is located at every heat station to maintain the pressure difference between supply and return network. The power consumption of the water pump at the heat source is given by

$$
P_{s}^{W P}=\frac{m_{s}^{H S}\left(p r_{n}^{S}-p r_{n}^{R}\right)}{\rho \eta^{W P}}, \forall n \in \Lambda^{N}, \forall s \in \Theta_{n}^{H S}
$$

and limited by its technical limits

$$
\underline{P}_{s}^{W P} \leq P_{s}^{W P} \leq \bar{P}_{s}^{W P}, \forall s \in \Theta_{n}^{H S}
$$

The heat demand is defined as

$$
H_{l}^{L}=C m_{l}^{H L}\left(T_{n}^{S}-T_{n}^{R}\right), \forall n \in \Lambda^{N}, \forall l \in \Theta_{n}^{H L}
$$

To ensure the flowing of water through the load, the pressure difference between the supply and return network at the load node has to be greater than a specified level:

$$
p r_{n}^{S}-p r_{n}^{R} \geq \underline{p r}_{l}^{H L}, \forall n \in \Lambda^{N}, \forall l \in \Theta_{n}^{H L}
$$

Heat losses are modeled through the temperature drop equation along the pipes:

$$
\begin{aligned}
& T_{p}^{S, \text { end }}-T_{a}=\left(T_{p}^{S, \text { start }}-T_{a}\right) e^{-\frac{\lambda L_{p}}{C m_{p}^{S}}}, \forall p \in \Lambda^{P} \\
& T_{p}^{R, \text { end }}-T_{a}=\left(T_{p}^{R, \text { start }}-T_{a}\right) e^{-\frac{\lambda L_{p}}{C m_{p}^{R}}}, \forall p \in \Lambda^{P}
\end{aligned}
$$

The temperature of the water leaving a node with more than one incoming pipe is calculated as the mixture temperature of the incoming flows.

$$
\begin{gathered}
\sum_{p \in \Theta_{n}^{P, I N}} m_{p}^{S} T_{p}^{S, \text { end }}=T_{n}^{S} \sum_{p \in \Theta_{n}^{P, I N}} m_{p}^{S}, \forall n \in \Lambda^{N} \\
\sum_{p \in \Theta_{n}^{P, \text { OUT }}} m_{p}^{R} T_{p}^{R, \text { end }}=T_{n}^{R} \sum_{p \in \Theta_{n}^{P, \text { OUT }}} m_{p}^{R}, \forall n \in \Lambda^{N}
\end{gathered}
$$

The starting temperature of the pipes are nodal temperatures:

$$
\begin{aligned}
& T_{n}^{S}=T_{p}^{S, \text { start }}, \forall n \in \Lambda^{N}, \forall p \in \Theta_{p}^{P, \text { OUT }} \\
& T_{n}^{R}=T_{p}^{R, \text { start }}, \forall n \in \Lambda^{N}, \forall p \in \Theta_{p}^{P, I N},
\end{aligned}
$$

For a node with a single pipe starting or ending, next relationships hold:

$$
\begin{aligned}
& T_{n}^{S}=T_{p}^{S, \text { end }}, \forall n \in \Lambda^{N-}, \forall p \in \Theta_{p}^{P, I N} \\
& T_{n}^{R}=T_{p}^{R, \text { end }}, \forall n \in \Lambda^{N+}, \forall p \in \Theta_{p}^{P, O U T}
\end{aligned}
$$

All the temperatures are withing their operational bounds:

$$
\begin{aligned}
& \underline{T}_{n}^{S} \leq T_{n}^{S} \leq \bar{T}_{n}^{S}, \underline{T}_{n}^{R} \leq T_{n}^{R} \leq \bar{T}_{n}^{R}, \forall n \in \Lambda^{N} \\
& \underline{T}_{p}^{S} \leq T_{p}^{S, \text { start/end }} \leq \bar{T}_{p}^{S}, \forall p \in \Lambda^{P} \\
& \underline{T}_{p}^{R} \leq T_{p}^{R, \text { start/end }} \leq \bar{T}_{p}^{R}, \forall p \in \Lambda^{P}
\end{aligned}
$$

For the extraction CHP unit the reserve capacity that could be activated in real-time is considered.

$$
\begin{aligned}
& \left(P_{s}^{C H P}-R_{s}^{d c}\right) \geq r_{s} H_{s}^{H S}+\underline{P}_{s}^{C H P}, \forall s \in \Theta_{n}^{C H P} \\
& 0 \leq \rho_{s}^{E}\left(P_{s}^{C H P}+R_{s}^{u c}\right)+\rho_{s}^{H} H_{s}^{H S} \leq \bar{F}, \forall s \in \Theta_{n}^{C H P}
\end{aligned}
$$

The heat output and power consumption of heat pumps are linked by the Coefficient of Performance

$$
H_{s}^{H S}=C O P_{s} P_{s}^{H P}, \forall s \in \Theta_{n}^{H P}
$$




\section{ELECTRICAL POWER SYSTEM MODEL}

To approximate nonconvex $\mathrm{AC}$ power flow equations, we use DC power flow approximation.

$$
\begin{gathered}
\mathbf{1}^{T} P^{G}+\mathbf{1}^{T} P^{C H P}+\mathbf{1}^{T} P^{f} \\
=\mathbf{1}^{T} P^{D}+\mathbf{1}^{T} P^{W P}+\mathbf{1}^{T} P^{H P} \\
-P^{l} \leq A\left[C(P+R)+C_{W}\left(P^{f}+\Delta W\right)-C_{D} P^{D}\right. \\
\left.-C_{W P} P^{W P}-C_{H P} P^{H P}\right] \leq P^{l} \\
-R T^{d n} \leq R \leq R T^{u p} \\
P^{m i n} \leq P+R \leq P^{\max } \\
0 \leq R^{u p} \leq \bar{R}^{G}, \quad 0 \leq R^{d n} \leq \underline{R}^{G}, \\
0 \leq R^{u c} \leq \bar{R}^{C H P}, \quad 0 \leq R^{d c} \leq \underline{R}^{C H P}, \\
R=-Y \sum_{w} \Delta W_{w}, \quad \sum_{i} Y=1, \quad Y \geq \mathbf{0}, \quad Y \leq \mathbf{1}
\end{gathered}
$$

where matrices $C_{G}, C_{C}, C_{W}, C_{D}, C_{W P}$, and $C_{H P}$ are mapping generators, CHPs, wind power plants, loads, water pumps, and heat pumps to their buses. $P=\left[P^{G} ; P^{C H P}\right]$ is a stacked column vector for the day-ahead dispatch and $C=\left[\begin{array}{ll}C_{G} & C_{C}\end{array}\right]$ - matrix that maps generators and CHPs with their buses. $\Delta W$ - is a vector of the uncertain wind forecast errors, which are revealed in real-time. Matrix A - is a power transfer distribution factor matrix. $R T^{u p}=\left[R^{u p} ; R^{u c}\right]$, $R T^{d n}=\left[R^{d n} ; R^{d c}\right]$ are stacked column vectors of the upward and downward reserves. $Y \in \mathbb{R}^{\left(N_{G}+N_{C H P}\right) \times 1}$ - is a vector of participation factors for controllable generators and CHP.

\section{A. Objecive function}

The objective function includes three terms: operational cost of generators, CHP units, and cost for reserves

$$
\begin{gathered}
C^{G}\left(P^{G}\right)+C^{C H P}\left(P^{C H P}, H^{H S}\right) \\
+C^{R}\left(R^{u p}, R^{d n}, R^{u c}, R^{d c}\right) \\
C^{G}\left(P^{G}\right)=\left(P^{G}\right)^{T} c_{2}^{T} P^{G}+c_{1}^{T} P^{G}+c_{0}^{T} \\
C^{C H P}\left(P^{C H P}, H^{H S}\right)=c_{e}^{T} P^{C H P}+c_{h}^{T} H^{C H P} \\
C^{R}\left(R^{u p}, R^{d n}, R^{u c}, R^{d c}\right)=\bar{c}_{G}^{T} R^{u p}+\underline{c}_{G}^{T} R^{d n}+\bar{c}_{c}^{T} R^{u c}+\underline{c}_{c}^{T} R^{d c}
\end{gathered}
$$

\section{Distributionally Robust ReFormulation}

In this section, the distributionally robust reformulation of the problem is introduced, which builds an ambiguity set to constrain the distribution of the uncertainty. This reformulation follows [12]. Stochastic constraints that involve random variable (19), (20), (21) could be written as one-side chanceconstraints

$$
\begin{gathered}
\mathbb{P}\left(A \left[C(P+R)+C_{W}\left(P^{f}+\Delta W\right)-C_{D} P^{D}\right.\right. \\
\left.\left.-C_{p} P^{W P}-C_{H P} P^{H P}\right] \geq-P^{l}\right) \geq 1-\epsilon \\
\mathbb{P}\left(A \left[C(P+R)+C_{W}\left(P^{f}+\Delta W\right)-C_{D} P^{D}\right.\right. \\
\left.\left.-C_{p} P^{W P}-C_{H P} P^{H P}\right] \leq P^{l}\right) \geq 1-\epsilon \\
\mathbb{P}(P+R \geq \underline{P}) \geq 1-\epsilon
\end{gathered}
$$

$$
\begin{gathered}
\mathbb{P}(P+R \leq \bar{P}) \geq 1-\epsilon \\
\mathbb{P}\left(R \leq R T^{u p}\right) \geq 1-\epsilon \\
\mathbb{P}\left(R \geq-R T^{d n}\right) \geq 1-\epsilon
\end{gathered}
$$

where $\epsilon$ - is the violation probability. By substituting $R=$ $-Y \sum_{w} \Delta W_{w}$ and the summation into $\sum_{w}=\mathbf{1}_{1 \times N_{W}}^{T}$ we can fraction out the uncertain part. Then the inner part of the above mentioned constraints can be reformulated as $\left(A_{i}^{x}\right)^{T} \xi \leq$ $b_{i}^{x}, \forall i=1, \ldots m$ where random variable $\xi$ is wind forecast error $\Delta W, m$ - is a number of chance-constraints, matrices $A_{i}^{x}$ and $b_{i}^{x}$ - deterministic parts of the constraints which depend only on the decision variables. The DR variants of chanceconstraints are formulated as follows

$$
\inf _{\mathbb{P}_{\xi} \in \mathcal{D}_{\xi}} \mathbb{P}\left(\left(A_{i}^{x}\right)^{T} \xi \leq b_{i}^{x}\right) \geq 1-\epsilon_{i}, \forall i=1 \ldots m
$$

After calculating from the data samples $\left\{\xi^{l}\right\}_{l=1}^{N}$ the empirical mean vector $\mu_{N_{W} \times 1}=\frac{1}{N} \sum_{l=1}^{N} \xi^{l}$ and covariance matrix $\Sigma_{N_{W} \times N_{W}}=\frac{1}{N} \sum_{l=1}^{N}\left(\xi^{l}-\mu\right)\left(\xi^{l}-\mu\right)^{T}$ we can build moment-based ambiguity set $\mathcal{D}_{\xi}:=\left\{\mathbb{P}_{\xi} \in \mathcal{P}^{\prime}: \mathbb{E}_{\mathbb{P}_{\xi}}[\xi]=\right.$ $\left.\mu, \mathbb{E}_{\mathbb{P}_{\xi}}\left[(\xi-\mu)(\xi-\mu)^{T}\right]=\Sigma\right\}$ which requires that the true mean and covariance matrix of $\xi$, given by any distribution in set $\mathcal{D}_{\xi}$, be exactly the empirical mean $\mu$ and covariance $\Sigma$. In [12], [16] it is shown that given the first and secondorder moment information for the constraint vectors, general DR chance-constraints can be reformulated as follows

$$
\sqrt{\left(\frac{1-\epsilon}{\epsilon}\right)\left(A_{i}^{x}\right)^{T}(\Sigma) A_{i}^{x}} \leq b_{i}^{x}-\left(A_{i}^{x}\right)^{T} \mu, \forall i=1 \ldots m
$$

which is a second-order cone constraint:

$$
K_{\epsilon}\left\|(\Sigma)^{\frac{1}{2}} A_{i}^{x}\right\|_{2} \leq b_{i}^{x}-\left(A_{i}^{x}\right)^{T} \mu, \forall i=1 \ldots m
$$

where $K_{\epsilon}=\sqrt{\left(\frac{1-\epsilon}{\epsilon}\right)}$. In this work, matrix $\Sigma$ considers spatial correlation.

As a result, the full optimization problem is

$$
\min (25)
$$

subject to: (1)-(18), (22)-(24) and (26) constraints, over the set of optimization variables

$$
\begin{aligned}
\Xi=\{ & m_{p}^{S}, m_{p}^{R}, m_{s}^{H S}, m_{l}^{H L}, \\
& T_{p}^{S, \text { start }}, T_{p}^{S, \text { end }}, T_{p}^{R, \text { start }}, T_{p}^{R, \text { end }}, T_{n}^{S}, T_{n}^{R}, H_{s}^{H S}, \\
& \left.P^{C H P}, P^{H P}, P^{W P}, P^{G}, R^{u p}, R^{u c}, R^{d n}, R^{d c}, Y\right\}
\end{aligned}
$$

\section{Convex Relaxation AND Linearization of the DHN EQUATIONS}

Mass flow rates under CF-VT control strategy are fixed. However, VF-VT control strategy has more practical meaning, and hence the model is non-convex and fully nonlinear because of the constraints (3), (5), (7), (9), (11) and (12). The comparison between two control strategies can be found in [9]. To guarantee the global optimum, the problem needs to be convex. One way is to use different relaxation approaches, which can provide a lower bound number on the optimal solution of the original problem. 


\section{A. McCormick Envelopes}

The most standard approach to handle bi-linear terms (5), (7), (9) and (12) is to apply a McCromick relaxation approach [17]. Each bilinear term $x y, x \in\left[x^{L}, x^{U}\right], y \in\left[y^{L}, y^{U}\right]$ is replaced with a new variable $w=x y$. Then two underestimators and two overestimators of the original term are introduced. McCormick envelopes of the DHN equations are detailed in the online appendix of [6].

\section{B. Convex quadratic relaxation}

The pressure loss constraint (3) is a quadratic term and relaxed into its convex envelope [18].

$$
\begin{aligned}
& p r_{n_{1}}^{S}-p r_{n_{2}}^{S} \geq K_{p}\left(m_{p}^{S}\right)^{2}, p r_{n_{1}}^{R}-p r_{n_{2}}^{R} \geq K_{p}\left(m_{p}^{R}\right)^{2}, \\
& \operatorname{pr}_{n_{1}}^{S}-p r_{n_{2}}^{S} \leq K_{p}\left(\left(\bar{m}_{p}^{S}+\underline{m}_{p}^{S}\right) m_{p}^{S}+\left(\bar{m}_{p}^{S}-\underline{m}_{p}^{S}\right)\right), \\
& p r_{n_{1}}^{R}-p r_{n_{2}}^{R} \leq K_{p}\left(\left(\bar{m}_{p}^{R}+\underline{m}_{p}^{R}\right) m_{p}^{R}+\left(\bar{m}_{p}^{R}-\underline{m}_{p}^{R}\right)\right), \\
& \forall p \in \Lambda^{P}, n_{1} \in \Theta_{n}^{P, O U T}, n_{2} \in \Theta_{n}^{P, I N}
\end{aligned}
$$

\section{Temperature loss equation linearizaton}

Constraint (11) can be written as

$$
\begin{aligned}
& T_{p}^{S, \text { end }}-T_{a}=\left(T_{p}^{S, \text { start }}-T_{a}\right) K_{p}^{\text {loss }}\left(m_{p}^{S}\right), \forall p \in \Lambda^{P} \\
& T_{p}^{R, \text { end }}-T_{a}=\left(T_{p}^{R, \text { start }}-T_{a}\right) K_{p}^{\text {loss }}\left(m_{p}^{R}\right), \forall p \in \Lambda^{P}
\end{aligned}
$$

$K_{p}^{\text {loss }}\left(m_{p}^{S}\right)$ for the pipes with relative high mass flow rates from $100 \mathrm{~kg} / \mathrm{s}$ is less that $0.1 \%$. Indeed, we can substitute the $K_{p}^{\text {loss }}\left(m_{p}^{S}\right)$ and $K_{p}^{\text {loss }}\left(m_{p}^{R}\right)$ with constants coefficients of mid-range of the mass flows.

As a result, the original problem is relaxed into a convex SOCP and can be solved by commercial solvers.

\section{Feasibility issue}

The solutions to the relaxed problem could be infeasible for the original model and relaxed constraints (3), (5), (7), (9), (11), (12) can be violated. In order to obtain feasible solutions, we solve the original problem with constant mass flow rates obtained from the relaxed problem.

\section{CASE STUdY}

\section{A. Test System}

The integrated six-bus electrical power system and sevennode district heating system is depicted in Fig. 1. The model is tested over 1 hour time step (single-period). Cost coefficients for CHP and the generator are taken from [4]. We assume that reserves from generator are more expensive than CHP reserves $\overline{c_{G}}=\underline{c_{G}}=1.2 c_{1}, \overline{c_{c}}=\underline{c_{c}}=1.1 c_{e}$

The characteristics of the generation units, transmission lines parameters and DHN parameters are detailed in [14]. Wind power output data for 6 wind farms in southeastern Australia from [19] is used. The samples are generated as in [13].

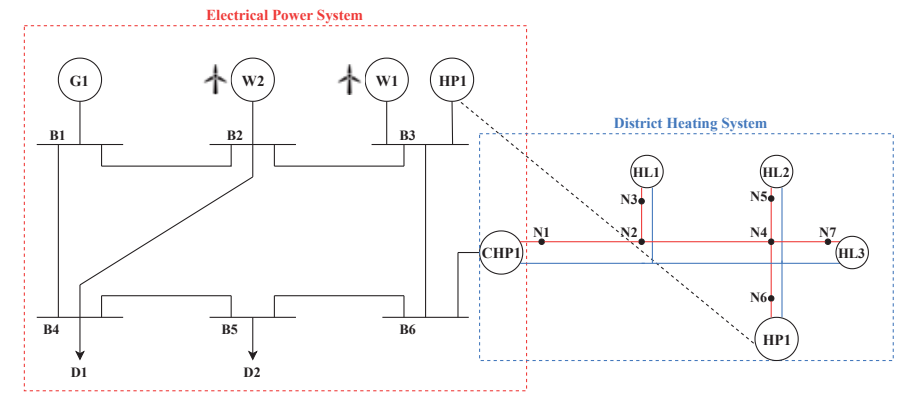

Fig. 1. Configuration of the six-bus and seven-node integrated system.

\section{B. Evaluation procedure}

The set up we used can be found in [12]. After solving the full model for a single-period, using an hour-ahead forecast, the optimal solution $x^{*}=\left[P^{*} ; Y^{*} ; P^{H P^{*}} ; P^{W P^{*}} ; H^{H S *}\right]$ is obtained and fixed. Next, these optimal decisions are evaluated and tested on $M=10000$ samples of wind power forecast error realization $\Delta \tilde{W}$. For every realization, $k=1 \ldots M$, the actual power output of the generator and CHP is computed such as

$$
P_{r t}^{*}(k)=P^{*}-Y^{*} \sum_{w} \Delta \tilde{W}_{w}(k)
$$

This gives us the actual power flow along the lines. Due to the linkage between heat and electricity, the power output of the CHP units is limited after the day-ahead heat is scheduled. Hence, for computing out-of-sample violation probabilities, the CHP power output limits are recomputed by taking into account the fixed scheduled day-ahead heat dispatch in (16). Then empirical out-of-sample violation probabilities are evaluated. We run 10 independent simulations and resolve the optimization problem 10 times. The model is solved by MOSEK [20] and implemented in Matlab with a CVX package [21].

\section{Results}

In [12], it is shown that under the assumption, that uncertainty is Gaussian, the problem can be reformulated as a convex problem. We report results for two cases: assuming the uncertainty is Gaussian and if the forecast error distribution is unknown i.e., distributionally robust case (DRO).

In Table I the objective function in $\$$ and CPU time in seconds are shown. The results demonstrate several things. First, it could be seen that the cost for the relaxed solution is always less than for the feasible solution. This complies with the fact that the relaxed solution is a lower bound for the original problem. Second, the CPU time is higher for the relaxed problem, because the relaxed problem has more constraints. Moreover, from the results, it is clear that Gaussian approximation obtains less cost than the distributional robust reformulation. This is consistent with what has been found in previous studies. It is also important to highlight that with the increment of the violation probability to $\epsilon=10 \%$, the cost decreased because he decisions are then less conservative and we allow constraints to be violated $90 \%$ times. 
TABLE I

COST PERFORMANCE AND CPU TIME

\begin{tabular}{ccccccc}
\hline \multirow{6}{*}{} & & \multicolumn{2}{c}{ Gaussian } & \multicolumn{2}{c}{ DRO } \\
\cline { 3 - 7 } & & $1-\epsilon$ & $95 \%$ & $90 \%$ & $95 \%$ & $90 \%$ \\
\hline \multirow{4}{*}{ Cost } & \multirow{4}{*}{ Relaxed } & avg & 5350.2 & 5161.8 & 8803.5 & 6983.5 \\
& & $\max$ & 5976.9 & 5761.0 & 12713.4 & 9417.7 \\
& & $\min$ & 4430.8 & 4265.2 & 5791.72 & 5048.9 \\
\cline { 3 - 7 } & Feasible & avg & 5975.9 & 5787.3 & 9429.8 & 7609.6 \\
& & $\max$ & 6603.1 & 6387.1 & 13339.8 & 10044.2 \\
& & $\min$ & 5055.7 & 4889.9 & 6417.6 & 5674.32 \\
\hline \multirow{4}{*}{ CPU } & \multirow{4}{*}{ Relaxed } & Feasible & 0.393 & 0.575 & 0.17 & 0.333 \\
& & $\max$ & 0.42 & 0.61 & 0.19 & 0.36 \\
\cline { 3 - 7 } & & $\operatorname{avg}$ & 0.323 & 0.487 & 0.145 & 0.292 \\
& & $\max$ & 0.34 & 0.52 & 0.16 & 0.33 \\
\cline { 3 - 7 } & Overall & $\operatorname{avg}$ & 0.71 & 1.062 & 0.315 & 0.625 \\
& & $\max$ & 0.77 & 1.11 & 0.33 & 0.67 \\
\hline
\end{tabular}

TABLE II

EMPIRICAL VIOLATION PROBABILITIES UNDER DIFFERENT DISTRIBUTION ROBUSTNESS

\begin{tabular}{cccccc}
\hline & & \multicolumn{2}{c}{ Gaussian } & \multicolumn{2}{c}{ DRO } \\
\cline { 3 - 6 } & $1-\epsilon$ & $95 \%$ & $90 \%$ & $95 \%$ & $90 \%$ \\
\hline \multirow{3}{*}{ Line vio. } & avg & 0 & 0 & 0 & 0 \\
& $\max$ & 0 & 0 & 0 & 0 \\
& $\min$ & 0 & 0 & 0 & 0 \\
\hline \multirow{3}{*}{ G1 vio. } & avg & 0 & 0 & 0 & 0 \\
& $\max$ & 0 & 0 & 0 & 0 \\
& $\min$ & 0 & 0 & 0 & 0 \\
\hline \multirow{3}{*}{ CHP1 vio. } & $\operatorname{avg}$ & $5.40 \%$ & $8.41 \%$ & $0.12 \%$ & $1.21 \%$ \\
& $\max$ & $46.02 \%$ & $52.28 \%$ & $2.42 \%$ & $21.32 \%$ \\
& $\min$ & 0 & 0 & 0 & 0 \\
\hline
\end{tabular}

From Table II we conclude that the assumption about Gaussian distribution of the uncertainty yields poor reliability and CHP feasible operating region is violated in $46.02 \%$ and $52.28 \%$ out of sample tests for the theoretical $\epsilon=5 \%$ and $\epsilon=10 \%$ respectively. The DRO case with $\epsilon=10 \%$ shows the maximum violation probability of $21.32 \%$. This number is more than expected and it indicates that the uncertainty needs to be considered in the DHN. That is to say, for the CHP to provide full flexibility, the heat power has to be redispatched in real-time. However, superior results are seen for the $\epsilon=5 \%$ case with maximum violation probability of the CHP feasible operation region $2.42 \%$. It is interesting to note that the violation probabilities for the generator operation limits and line limits capacity are 0 .

Figure 2 reports scheduled energy and reserves. The plotted values are averaged over 10 simulations. The reserves are the sum of the downward and upward reserves. The averaged forecasted wind power in the system $P_{w 1}^{f}=19.35 \mathrm{MW}$ and $P_{w 2}^{f}=23.35 M W$. We observe that the Gaussian case scheduled reserves capacity only from the CHP. The reason for this is clear. This demonstrates the fact that Gaussian reformulation relies only on less costly CHP reserves. This

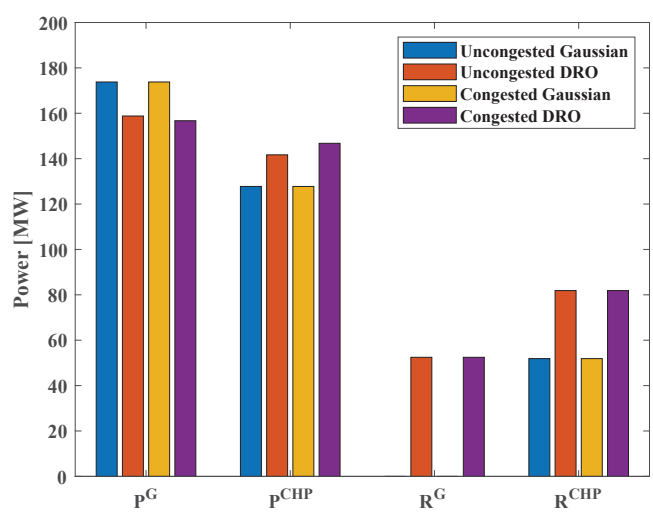

Fig. 2. Optimal reserve procurement and generation dispatch, $\epsilon=5 \%$.

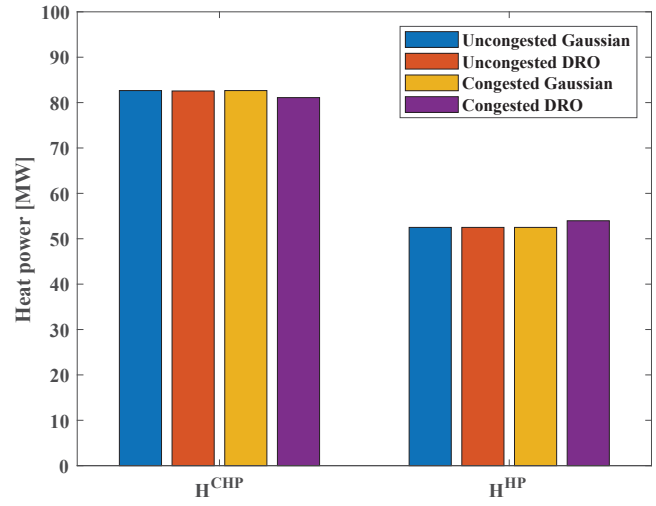

Fig. 3. Optimal heat dispatch.

TABLE III

RESERVE PROCUREMENT, $\epsilon=5 \%$

\begin{tabular}{cccc}
\hline & & Gaussian & DRO \\
\hline \multirow{3}{*}{ Congested } & Generator Reserves & $7.7487 \mathrm{e}-06$ & 52.47 \\
\cline { 2 - 4 } & CHP reserves & 51.89 & 81.87 \\
\cline { 2 - 4 } & Total Reserves & 51.89 & 134.34 \\
\hline \multirow{3}{*}{ Uncongested } & Generator Reserves & $7.7487 \mathrm{e}-06$ & 52.47 \\
\cline { 2 - 4 } & CHP reserves & 51.89 & 81.87 \\
\cline { 2 - 4 } & Total Reserves & 51.89 & 134.34 \\
\hline
\end{tabular}

could be an explanation of why the violation probabilities are much more higher than in the DRO case. However, the DRO approach "cares" more about reliability and schedules more costly reliable generator reserves.

Figure 3 shows averaged over the 10 simulations heat dispatch from the CHP and heat pump. We can note that the choice of the method does not affect the heat dispatch. The total amount reveres procured for the congested and uncontested case can be seen from Table III. For each approach, the amount of total reserves is the same in the congested and the uncontested cases. This confirms previous findings in [12].

Finally, the hydraulic and thermal profiles of the district 


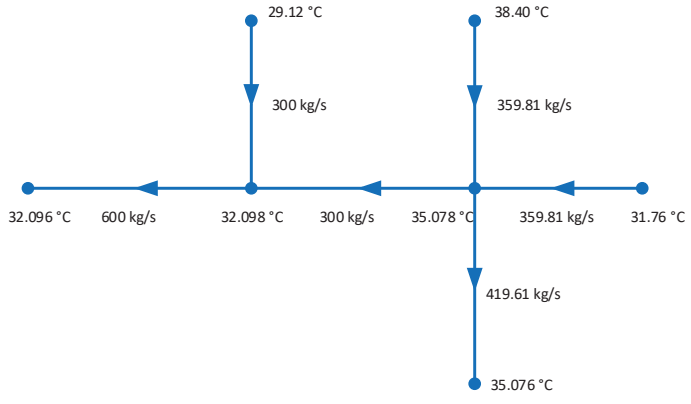

Fig. 4. Mass flow and thermal profile. Return network

heating system are presented Fig. 4. The mass flows are depicted below the pipes, and the nodal temperatures are close to the nodes. As it was observed before the temperatures drop along the pipes due to the heat transfer in the supply and the return network is withing thousandth. Return temperature at node 4 is higher than at node 7 since this is the mix temperatures of the incoming flows from nodes 5 and 7 . The same conclusion applies to nodes 2 and 3 . The lowest return temperature is at node 3 due to the lowest mass flow rate at pipe 2 .

\section{CONCLUSION}

This paper has investigated the distributionally robust approach of scheduling day-ahead energy and reserve capacity in integrated electricity and heating systems considering the district heating network. Tests of the model have been conducted on the small case study in Section VI-C in a data-driven manner. The results of this study indicate that the method could be successfully applied in the optimal operation of the integrated electricity and heating systems in order to deal with the increased uncertainty from renewable energy sources. The system is operated reliably without re-dispatching heat power in real-time with a confidence level of $95 \%$. However, the wind power uncertainty has to be propagated from the power to the heat side, for operating the system at $90 \%$ confidence level.

Future work will investigate the possible application of this model for the multi-time period dispatch (e.g., 24 h) with considering uncertainty in the heat side and wind power temporal correlation [22]. Moreover, to explore the full flexibility of the DHN, the reserves from the flexible loads in the DHN (e.g., a heat pump) could be provided with the framework of [12]. Furthermore, the performance of the model needs to be tested on a bigger case study with the real data.

\section{ACKNOWLEDGMENT}

This work was supported in part by the EUDP programme through the SMART MLA project (grant no 64018-0302) and in part by DTU through the Alliance PhD scholarship.

\section{REFERENCES}

[1] P. Veers, K. Dykes, E. Lantz, S. Barth, C. L. Bottasso, O. Carlson, A. Clifton, J. Green, P. Green, H. Holttinen, D. Laird, V. Lehtomäki, J. K. Lundquist, J. Manwell, M. Marquis, C. Meneveau, P. Moriarty,
X. Munduate, M. Muskulus, J. Naughton, L. Pao, J. Paquette, J. Peinke, A. Robertson, J. Sanz Rodrigo, A. M. Sempreviva, J. C. Smith, A. Tuohy, and R. Wiser, "Grand challenges in the science of wind energy," Science, vol. 366 , no. $6464,2019$.

[2] "Danish climate policies: Targets impacting danish climate policies." [Online]. Available: https://ens.dk/en/our-responsibilities/ energy-climate-politics/danish-climate-policies

[3] H. Lund, B. Möller, B. V. Mathiesen, and A. Dyrelund, "The role of district heating in future renewable energy systems," Energy, vol. 35, no. 3, pp. 1381-1390, 2010.

[4] Z. Li, W. Wu, M. Shahidehpour, J. Wang, and B. Zhang, "Combined heat and power dispatch considering pipeline energy storage of district heating network," IEEE Transactions on Sustainable Energy, vol. 7, no. 1, pp. 12-22, 2015.

[5] J. Li, J. Fang, Q. Zeng, and Z. Chen, "Optimal operation of the integrated electrical and heating systems to accommodate the intermittent renewable sources," Applied Energy, vol. 167, pp. 244-254, 2016.

[6] L. Mitridati and J. A. Taylor, "Power systems flexibility from district heating networks," 20th Power Systems Computation Conference, PSCC 2018, pp. 1-7, 2018.

[7] S. Huang, W. Tang, Q. Wu, and C. Li, "Network constrained economic dispatch of integrated heat and electricity systems through mixed integer conic programming," Energy, vol. 179, pp. 464-474, 2019.

[8] M. Bornapour, R.-A. Hooshmand, A. Khodabakhshian, and M. Parastegari, "Optimal coordinated scheduling of combined heat and power fuel cell, wind, and photovoltaic units in micro grids considering uncertainties," Energy, vol. 117, pp. 176-189, 2016.

[9] Z. Li, W. Wu, J. Wang, B. Zhang, and Z. Taiyi, "TransmissionConstrained Unit Commitment Considering Combined Electricity and District Heating Networks," IEEE Transactions on Sustainable Energy, vol. 7, no. 2, pp. 480-492, 2016.

[10] C. Shang and F. You, "Distributionally robust optimization for planning and scheduling under uncertainty," Computers \& Chemical Engineering, vol. 110 , pp. 53-68, 2018.

[11] M. Lubin, Y. Dvorkin, and S. Backhaus, "A robust approach to chance constrained optimal power flow with renewable generation," IEEE Transactions on Power Systems, vol. 31, no. 5, pp. 3840-3849, 2015.

[12] Y. Zhang, S. Shen, and J. L. Mathieu, "Distributionally robust chanceconstrained optimal power flow with uncertain renewables and uncertain reserves provided by loads," IEEE Transactions on Power Systems, vol. 32, no. 2, pp. 1378-1388, 2016.

[13] C. Ordoudis, V. A. Nguyen, D. Kuhn, and P. Pinson, "Energy and reserve dispatch with distributionally robust joint chance constraints," Technical University of Denmark, Tech. Rep., 2018.

[14] M. Skalyga, "Distributionally robust co-optimization of energy and reserve dispatch of integrated electricity and heat system - online appendix,” 2019. [Online]. Available: https://github.com/MikhailSkl/ DRO-IEHS-Online-Appendix.git

[15] X. Liu, "Combined analysis of electricity and heat networks," Ph.D. dissertation, Cardiff University, 2016.

[16] M. R. Wagner, "Stochastic 0-1 linear programming under limited distributional information," Operations Research Letters, vol. 36, no. 2, pp. $150-156,2008$.

[17] G. P. McCormick, "Computability of global solutions to factorable nonconvex programs: Part I - Convex underestimating problems," Mathematical Programming, vol. 10, no. 1, pp. 147-175, 1976.

[18] H. Hijazi, C. Coffrin, and P. V. Hentenryck, "Convex quadratic relaxations for mixed-integer nonlinear programs in power systems," Mathematical Programming Computation, vol. 9, no. 3, pp. 321-367, 2017.

[19] J. Dowell and P. Pinson, "Very-short-term probabilistic wind power forecasts by sparse vector autoregression," IEEE Transactions on Smart Grid, vol. 7, no. 2, pp. 763-770, 2015.

[20] M. ApS, "Mosek," 2019. [Online]. Available: https://www.mosek.com/

[21] M. Grant and S. Boyd, "CVX: Matlab software for disciplined convex programming, version 2.1," http://cvxr.com/cvx, Mar. 2014.

[22] X. Fang, B.-M. Hodge, E. Du, N. Zhang, and F. Li, "Modelling wind power spatial-temporal correlation in multi-interval optimal power flow: A sparse correlation matrix approach," Applied energy, vol. 230, pp. 531-539, 2018 